\title{
An expression signature of the angiogenic response in gastrointestinal neuroendocrine tumours: correlation with tumour phenotype and survival outcomes
}

\author{
D J Pinato ${ }^{1}$, T M Tan ${ }^{2}$, S T K Toussi ${ }^{1}$, R Ramachandran ${ }^{2}, \mathrm{~N} \mathrm{Martin}^{2}, \mathrm{~K} \mathrm{Meeran}^{2}, \mathrm{~N} \mathrm{Ngo}{ }^{3}, \mathrm{R} \mathrm{Dina}^{3}$ and \\ R Sharma*,1 \\ ${ }^{1}$ Division of Experimental Medicine, Imperial College London, Hammersmith Hospital, Du Cane Road, W120HS London, UK; \\ ${ }^{2}$ Department of Endocrinology, Imperial College London, Hammersmith Campus, Du Cane Road, W12OHS London, UK and \\ ${ }^{3}$ Department of Pathology, Imperial College London, Hammersmith Campus, Du Cane Road, W120HS London, UK
}

Background: Gastroenteropancreatic neuroendocrine tumours (GEP-NETs) are heterogeneous with respect to biological behaviour and prognosis. As angiogenesis is a renowned pathogenic hallmark as well as a therapeutic target, we aimed to investigate the prognostic and clinico-pathological role of tissue markers of hypoxia and angiogenesis in GEP-NETs.

Methods: Tissue microarray (TMA) blocks were constructed with 86 tumours diagnosed from 1988 to 2010 . Tissue microarray sections were immunostained for hypoxia inducible factor $1 \alpha$ (Hif-1 $\alpha$ ), vascular endothelial growth factor-A (VEGF-A), carbonic anhydrase IX (Ca-IX) and somatostatin receptors (SSTR) 1-5, Ki-67 and CD31. Biomarker expression was correlated with clinicopathological variables and tested for survival prediction using Kaplan-Meier and Cox regression methods.

Results: Eighty-six consecutive cases were included: 51\% male, median age 51 (range 16-82), 68\% presenting with a pancreatic primary, 95\% well differentiated, 51\% metastatic. Higher grading $(P=0.03)$, advanced stage $(P<0.001)$, high Hif- $1 \alpha$ and low SSTR-2 expression ( $P=0.03$ ) predicted for shorter overall survival $(O S)$ on univariate analyses. Stage, SSTR-2 and Hif- $1 \alpha$ expression were confirmed as multivariate predictors of OS. Median OS for patients with SSTR-2 + /Hif- $1 \alpha$-tumours was not reached after median follow up of 8.8 years, whereas SSTR-2-/Hif-1 $\alpha+$ GEP-NETs had a median survival of only 4.2 years $(P=0.006)$.

Conclusion: We have identified a coherent expression signature by immunohistochemistry that can be used for patient stratification and to optimise treatment decisions in GEP-NETs independently from stage and grading. Tumours with preserved SSTR-2 and low Hif- $1 \alpha$ expression have an indolent phenotype and may be offered less aggressive management and less stringent follow up.

Gastroenteropancreatic neuroendocrine tumours (GEP-NETs) are tumours of unknown aetiology with heterogeneous biological behaviour and prognosis. Although initially thought to be uncommon, the incidence and prevalence of NETs are increasing at a rate of $3-10 \%$ per year (Yao et al, 2008). The majority of patients with NETs present with metastatic disease, the commonest site being the liver. However, unlike all other gastrointestinal malignancies, patients with stage IV disease may enjoy fairly extended overall survival (OS) times, with median OS times approaching 33 months (Yao et al, 2008).

The prognostic assessment of GEP-NETs can be further refined by the use of the World Health Organization (WHO)

*Correspondence: Dr R Sharma; E-mail: r.sharma@imperial.ac.uk

Received 15 July 2013; revised 30 September 2013; accepted 8 October 2013; published online 14 November 2013

(c) 2014 Cancer Research UK. All rights reserved 0007-0920/14 
tumour grading system, which sub-classifies patients according to the degree of histological differentiation based on the proliferating fraction of NET cells (Hirabayashi et al, 2013). However, the natural biology of some GEP-NETs is such that there is significant variation in prognosis within each stage and grade subclass such that a proportion of patients will have a relative indolent course with a long OS in the absence of any clinical intervention. These patients may therefore receive treatment unnecessarily, being exposed to significant side effects without therapeutic benefit. Therefore, there is a need for more accurate prognostic markers in order individualise therapy in this otherwise very heterogeneous disease. Angiogenesis, the formation of new blood vessels from existing vasculature, is crucial in tumour progression both in terms of local invasion and metastatic spread (Hanahan and Weinberg, 2000). Normal endocrine tissues and endocrine-derived tumours are highly vascular, and numerous studies have shown a pathogenic role for the overexpression of vascular endothelial growth factor (VEGF) and other specific endothelial cell mitogens such as endocrine gland-derived VEGF (EG-VEGF) in these tumours (LeCouter et al, 2001; Katoh, 2003). The hypervascular nature of GEP-NETs has been exploited as a therapeutic target, and the clinical effectiveness of sunitinib, which in part relates to its antiangiogenic properties, has encouraged the clinical investigation of novel anti-angiogenic agents in the management of these tumours (Raymond et al, 2011).

Although the therapeutic relevance of angiogenesis is established in GEP-NETs, inconsistent results have emerged as to its prognostic role, mainly as previous studies have considered individual angiogenic markers as predictors of outcome rather than investigating the interplay between the various molecular actors that drive the hypoxic response (Chi et al, 2006). Although the adverse prognostic value of enhanced VEGF expression has been shown in some studies (Zhang et al, 2007), others report conflicting results with higher microvascular density and VEGF expression predicting for a more favourable outcome (Couvelard et al, 2005). In cancer, hypoxia inducible factors (Hif) regulate the expression of a number of genes that stimulate tumour cell proliferation in conditions of low oxygen tension, ultimately promoting angiogenesis, local invasion and metastasis. Under normal conditions, the Hif- $1 \alpha$ subunit is hydroxylated by proline hydroxylase in a reaction requiring oxygen and undergoes ubiquitination by the von Hippel-Lindau (VHL) protein and is then rapidly degraded in the proteasome (Giatromanolaki and Harris, 2001). Under conditions of hypoxia, Hif- $1 \alpha$ cannot be degraded and accumulates in the nucleus, leading to enhanced transcription of numerous hypoxic-response genes, such as VEGF, EG-VEGF and carbonic anhydrase IX (Ca-IX) (Hui et al, 2002; Maynard and Ohh, 2005).

Somatostatin receptors (SSTR) have been recognised as dynamic regulators of tumour development, cell proliferation and apoptosis (Ruscica et al, 2012). Somatostatin secretion is known to negatively influence VEGF production (Mentlein et al, 2001), and in vitro work has shown that the administration of the somatostatin analogue octreotide can antagonise Hif- $1 \alpha$ transcriptional activity in NET cells (Villaume et al, 2010). Moreover, SSTR-2 expression has been shown to have an anti-angiogenic role in animal models of hypoxia (Dal Monte et al, 2007), suggesting an active interplay between the somatostatin-signalling network and sustained angiogenesis.

We designed this study to investigate the prognostic role of a panel of immunohistochemical markers of hypoxia and angiogenesis using tissue microarray (TMA) technology on a consecutive series of patients presenting with GEP-NETs. We compared the prognostic utility of these markers with other more established prognostic determinants including tumour staging and grading.

\section{MATERIALS AND METHODS}

Patient population. A total of 88 consecutive patients who underwent surgical treatment for GEP-NETs at Imperial College NHS Trust from 1988 to 2010 were included. Formalin fixed, paraffin-embedded specimens and matching haematoxylin and eosin (H\&E) slides were retrieved from the local pathology archive.

Complete clinical and follow-up information including patient's demographics, tumour size and stage at diagnosis, site of presentation were retrieved by review of medical records. The presence of adverse pathological features such as vascular invasion and necrosis were identified following $\mathrm{H} \& \mathrm{E}$ slides review by a board certified endocrine pathologist (RD). Overall (cancer-specific) survival was calculated from the time of surgery to the time of death or last follow-up appointment. The study was approved by the local Ethical Committee and conducted in accordance to the principles of the Declaration of Helsinki. Serum levels of chromogranin $\mathrm{A}(\mathrm{CgA})$ at diagnosis was retrieved whenever available.

Tissue microarrays and immunohistochemistry. After review of $\mathrm{H} \& \mathrm{E}$-stained sections, three $1 \mathrm{~mm}$ cores were identified from the most representative areas of the tumour tissue, then re-embedded into recipient TMA blocks using an MTA-1 Manual Tissue Microarrayer (Beecher Instruments, Sun Prairie, WI, USA). Five$\mu$ m-thick TMA sections were deparaffinised in xylene and rehydrated in graded alcohols. Optimal heat-mediated antigen retrieval conditions were applied according to the primary antibody using a microwave oven at $900 \mathrm{~W}$. Incubation in citrate buffer at pH 6.0 for $20 \mathrm{~min}$ was preferred for antibodies VEGF-A (Santa Cruz Biotechnology, Santa Cruz, CA, USA), Hif-1 $\alpha$ (Abcam, Cambridge, UK), SSTR- 1-5 (Gramsch Laboratories, Schwabhausen, Germany) CD31 and Ki-67 (Leica Microsystems, Wetzlar, Germany), whereas antibodies to Ca-IX (Novus Biologicals, Littleton, CO, USA) were incubated in $\mathrm{pH} 9.0$ EDTA buffer for $30 \mathrm{~min}$. Before immunostaining, slides were cooled at room temperature and endogenous peroxidase activity was suppressed by incubation with a $3 \%$ solution of $\mathrm{H}_{2} \mathrm{O}_{2}$ for $5 \mathrm{~min}$. The primary antibody against Ki-67 was diluted to $1: 800$, whereas all the other antibodies were used at a $1: 1000$ dilution (Pinato et al, 2012b).

Tissue microarray slides were then stained through a fully automated procedure on a Bond Max Autostainer (Leica Microsystems) using the Polymer-HRP system (BioGenex, Fremont, CA, USA) with subsequent development in diaminobenzidine and Mayer's haematoxylin counterstaining. Appropriately selected tissue sections were used according to the manufacturer's instruction as external positive control during each reaction. Negative control reactions were performed omitting the primary antibodies from the dilution buffer. This resulted in a complete absence of staining in all cases. Two trained histopathologists ( $\mathrm{RD}$ and $\mathrm{NN}$ ) blinded to the clinical data scored all the cases and a consensus score was reached in any case of discrepancy. Tissue samples were scored manually using the immunohistochemical score method (IHS), as described previously (Pinato et al, 2012b). Briefly, each sample can be assigned an IHS ranging between 0 and 300 , based on the multiplication of the percentage of cells showing immunohistochemical expression (0-100) by the intensity of the signal (graded 1-3). Every core was assessed individually and the mean of the three readings was calculated per every single case. The pattern of staining (cytoplasmic, membranous, nuclear and diffuse) was also described in each case.

Statistical analysis. The association between IHS and clinicalpathological data of the patient cohort were determined by means of $\chi^{2}$, Fisher's exact test or one-way ANOVA as appropriate. For the purpose of analysis, the expression of each biomarker was categorised as high vs low based on the median value of the 
distribution of IHS across the whole study population. The Ki-67labelling index was expressed as the fraction of positive nuclei examining a minimum of 500 nuclei in at least five microscopic fields as previously described (Pinato et al, 2012a). The KaplanMeier statistics and Log-rank test were used to study the impact of the different clinical factors associated with OS on univariate analysis, with significant variables $(P<0.05)$ being further tested on a multivariate stepwise backward Cox regression model to validate their independent prognostic value. An entry threshold of 0.05 was used at each step of the multivariate analysis, whereas variables with a $P$-value $>0.10$ were removed from the model. Variables emerging as independent predictors of survival at the last step of the multivariate model were taken as significant if the correspondent $P$-value was $<0.10$. For all the other statistical tests a $P$-value $<0.05$ was taken to be significant. All statistical analysis was conducted using SPSS statistical package 11.5 (SPSS Inc., Chicago, IL, USA).

\section{RESULTS}

Clinico-pathological profile of patients. The baseline patient characteristics are shown in Table 1. Eighty-eight patients were identified. Clinical data were missing on two patients and they were excluded from subsequent analysis. The majority of clinical samples included in our study were composed of primary NET specimens $(76 / 86,88 \%)$, followed by seven liver metastatic deposits and three lymph node metastases. All patients had undergone radical resection of their GEP-NETs including excision of the primary tumour associated with concurrent lymphadenectomy and/or partial hepatectomy according to stage. The median age of the patients at the time of diagnosis was 51 years (range 16-82). The majority of patients were male $(53 \%)$, and had a primary pancreatic NET $(n=55), 34 \%$ of which were functional. Functional tumours were mainly insulinomas (27\%). Tumours were largely sporadic, with only five cases emerging in the context of Multiple Endocrine Neoplasia type 1. Forty-five patients (53\%) presented with metastatic disease, the commonest site of metastases being the liver $(73 \%)$ followed by locoregional spread to the lymph nodes (27\%). The majority of cases had well-differentiated tumours (95\%). The median tumour size was $30 \mathrm{~mm}$ (range 6-125 mm). Necrosis was present in $12(17 \%)$ of specimens and vascular invasion in 20 (23\%). Nineteen patients (22\%) had a Ki-67labelling index $>1 \%$.

World Health Organization grading was obtained in all cases and was scored as G1 in $84 \%$, G2 in $13 \%$ and G3 in $4 \%$. The circulating plasma level of $\mathrm{CgA}$ at the time of diagnosis was available for 27 patients (31\%), with mean value of $178 \mu \mathrm{gl} l^{-1}$. Plasma CgA levels $>100 \mu \mathrm{gl}{ }^{-1}$ correlated with the presence of liver metastases $(P=0.02)$. No association was observed between baseline $\mathrm{CgA}$ levels and survival. The median OS at the time of analysis was 8.8 years (range $0.04-13.5$ years).

Immunostaining in GEP-NETs and survival. The expression of the studied biomarkers is summarised in Table 1. The pattern of immunostaining was predominantly diffuse (cytoplasmic and nuclear) for Hif- $1 \alpha$, cytoplasmic for VEGF-A and membranous for SSTR-2 and Ca-IX (Figure 1). Somatostatin receptor-5 expression was cytoplasmic, whereas SSTR-1 was predominantly nuclear and SSTR-3 was diffuse. The median IHS value for Hif- $1 \alpha$ was 200 (range $0-300$ ), with elevated Hif- $1 \alpha$ expression being strongly associated with VEGF-A immunopositivity $(P<0.001)$. Median VEGF-A IHS was 240 (range $0-300$ ), whereas median Ca-IX IHS was 36 (0-300). High tissue expression levels of Hif- $1 \alpha$ and VEGF-A were associated with the presence of liver metastases both from the pancreatic and gut primaries $(P<0.05)$.
Table 1. General characteristics of the patient population

\begin{tabular}{|l|l|}
\hline Baseline characteristic & $\mathbf{n = 8 6}(\%)$ \\
\hline Gender & \\
\hline Male & $46(53)$ \\
Female & $40(47)$ \\
\hline
\end{tabular}

\begin{tabular}{|l|l|}
\hline Age, years \\
\hline$<55$ & $48(56)$ \\
$\geqslant 55$ & $38(44)$
\end{tabular}

Primary site

\begin{tabular}{|l|l|}
\hline Midgut & $31(36)$ \\
Pancreas & $55(64)$
\end{tabular}

Tumour size, $\mathrm{cm}$

\begin{tabular}{|l|c}
\hline$<3.0$ & $54(63)$ \\
$\geqslant 3.0$ & $28(32)$ \\
Unknown & $4(5)$ \\
\hline
\end{tabular}

\section{Stage}

Limited disease

Loco regional lymph nodal spread

Metastatic disease

Unknown

40 (47)

$12(14)$

$33(39)$

1 (1)

Functional status

\begin{tabular}{|l|c}
\hline Non-functioning & $56(65)$ \\
Functioning & $30(35)$ \\
Insulinoma & $23(27)$ \\
Gastrinoma & $7(8)$ \\
\hline
\end{tabular}

\section{Tumour necrosis}

\begin{tabular}{|l|c|}
\hline Absent & $70(78)$ \\
Present & $12(17)$ \\
Unknown & $4(5)$ \\
\hline
\end{tabular}

\begin{tabular}{|l|c|}
\hline \multicolumn{2}{|l|}{ Angioinvasion } \\
\hline Absent & $62(72)$ \\
Present & $20(23)$ \\
Unknown & $4(5)$
\end{tabular}

\begin{tabular}{|c|c|}
\hline \multicolumn{2}{|c|}{ Chromogranin A at presentation } \\
\hline $\begin{array}{l}<100 \mu \mathrm{gl}^{-1} \\
>100 \mu \mathrm{II}^{-1} \\
\text { Unknown }\end{array}$ & $\begin{array}{c}20(23) \\
7(8) \\
59(69)\end{array}$ \\
\hline
\end{tabular}

Grading (WHO 2010 criteria)

\begin{tabular}{|l|c|}
\hline Low & $72(84)$ \\
Intermediate & $11(13)$ \\
High & $3(3)$
\end{tabular}

SSTR expression, low/high

\begin{tabular}{|l|c|}
\hline SSTR-1 & $75 / 11(87 / 13)$ \\
SSTR-2 & $61 / 25(71 / 29)$ \\
SSTR-3 SSTR-4 & $84 / 2(98 / 2)$ \\
SSTR-5 & $86 / 0(100 / 0)$ \\
\hline \multicolumn{2}{|l|}{ Angiogenesis biomarker expression, low/high } \\
\hline Hif-1 $\alpha$ \\
VEGF-A & $41 / 45(100 / 0)$ \\
Carbonic anhydrase IX & $24 / 62(28 / 72)$ \\
CD31 & $77 / 9(89 / 11)$ \\
\hline \multicolumn{2}{|l}{$86 / 0(100 / 0)$} \\
Abbreviations: Ca-IX=carbonic anhydrase IX; Hif- $1 \alpha=$ hypoxia inducible factor alpha; \\
SSTR= somatostatin receptor; VEGF =vascular endothelial growth factor-A; WHO =World \\
Health Organization. \\
\hline
\end{tabular}



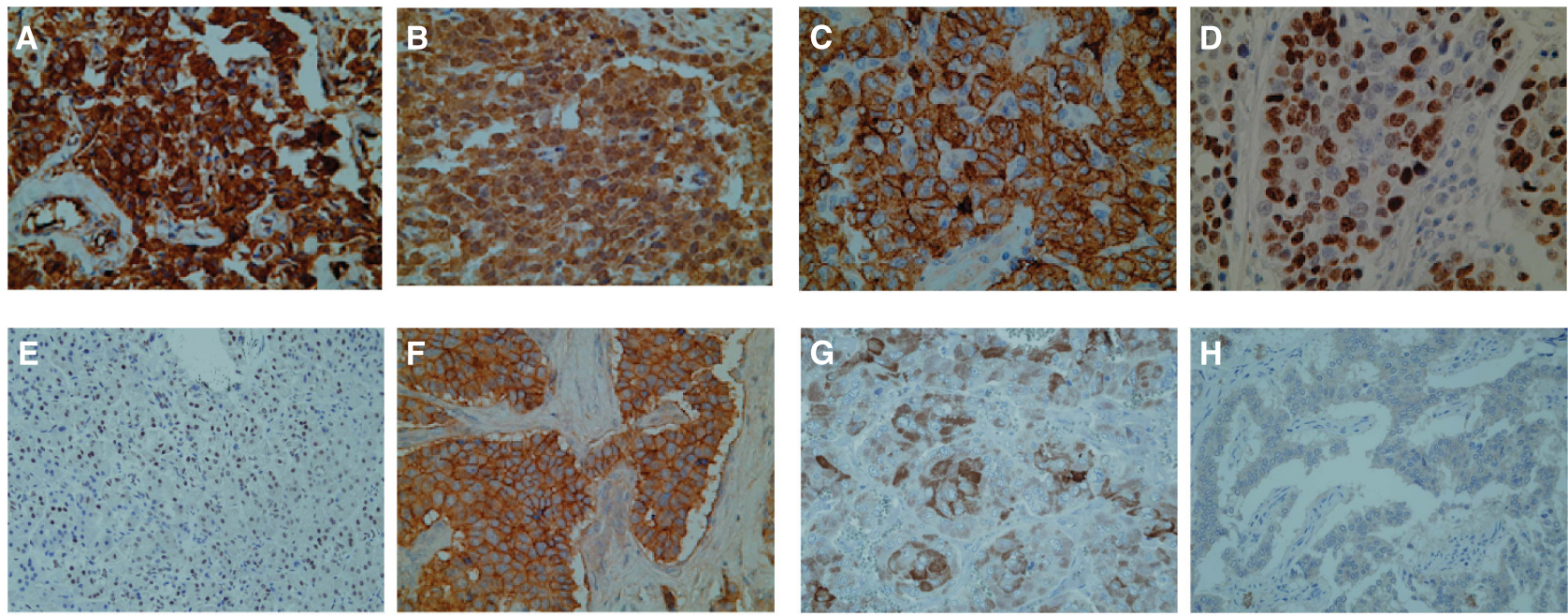

Figure 1. Expression of the candidate biomarkers in GEP-NETs by immunohistochemistry. Representative sections of tumours showing positive cytoplasmic staining for VEGF-A (A) and Hif-1 $\alpha$ (B) showing a typical diffuse granular expression pattern. (C) Illustrates strong Ca-IX expression within the neuroendocrine tumour cell membrane. (D) Shows a case of positive Ki-67 immunostaining in a high-grade neuroendocrine tumour. (E-H) show somatostatin receptor expression in GEPNETs, with a typical nuclear pattern in SSTR-1 (E), a strong membranous expression for SSTR-2 (F) followed by a predominant cytoplasmic immunolabelling for SSTR-3 and SSTR-5 expressions seen in (G and H), respectively. Magnification $\times 400$.
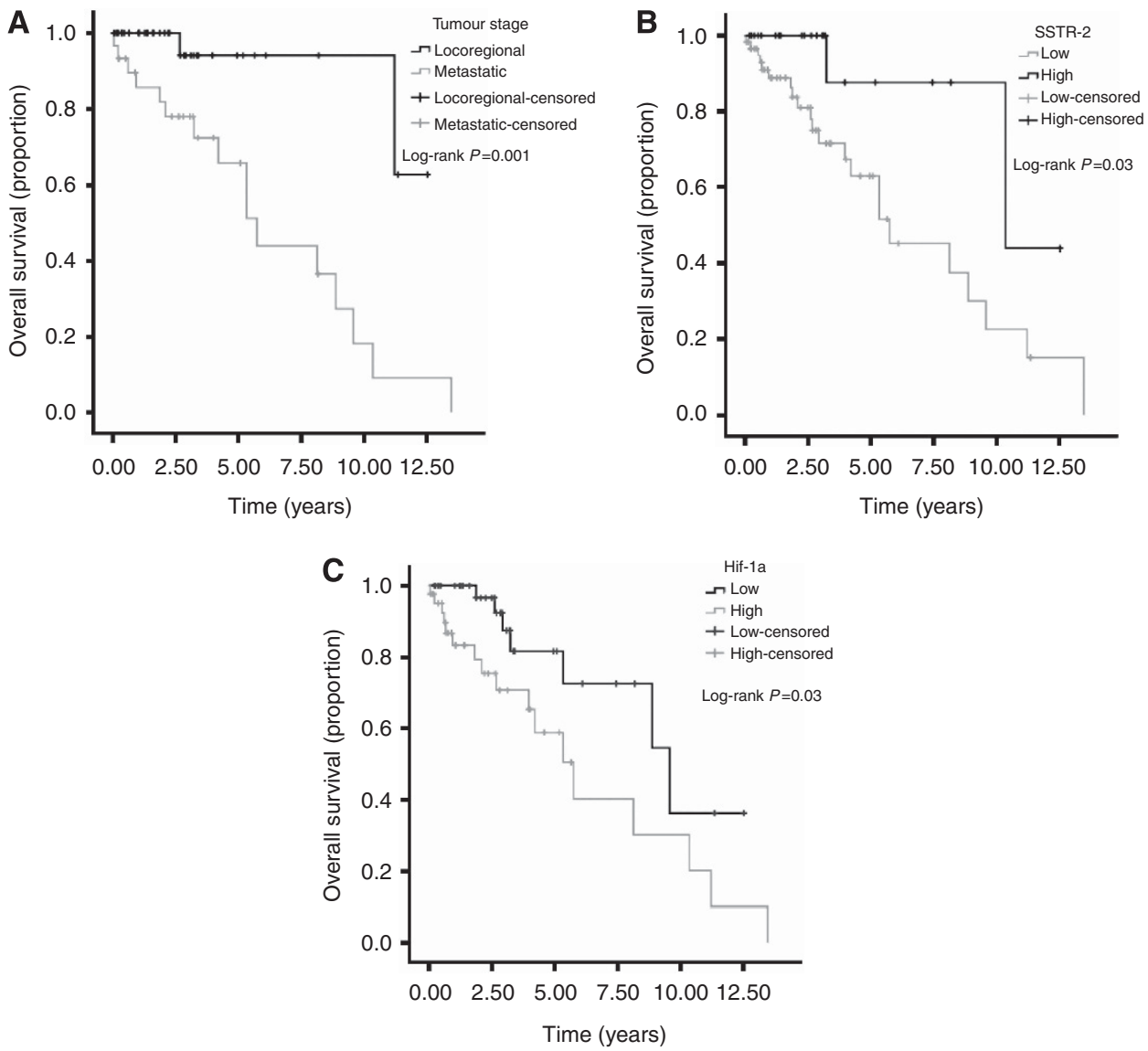

Figure 2. Kaplan-Meier curve analysis showing that advanced tumour stage (A), lack of SSTR-2 expression (B) and high Hif- $1 \alpha$ expression (C) predict for shortened OS in GEPNETs.

The median IHS for SSTR-1 was 9 (range 0-300), SSTR-2 median 100 (range 0-300), SSTR-3 0 (range 0-240) and SSTR-5 0 (range 0-90). The entire case series profiled was negative for both CD31 and SSTR-4 expressions.
In terms of the differing tumour types, pancreatic tumours demonstrated a higher expression of SSTR-2 $(P<0.01)$ and a trend was observed for higher expression of SSTR-1 $(P=0.06)$. Hypoxia inducible factor $1 \alpha$ was more highly expressed in carcinoid 
tumours compared with pancreatic tumours $(P=0.05)$. In terms of tumour characteristics, a significant association was observed between KI-67 and tumour size $(P<0.001)$ and the presence of necrosis $(P=0.03)$.

The prognostic value of each biomarker was tested together with established prognostic factors. Univariate analyses revealed the following variables predicted for poor OS: advanced tumour stage $(P=0.007)$ (Figure $2 \mathrm{~A})$, high WHO grading $(P=0.03)$, SSTR-2 expression loss $(P=0.03)$ and Hif- $1 \alpha$ overexpression $(P=0.03)$. Multivariate analysis of survival confirmed tumour stage $(P=0.008)$, low SSTR-2 $(P=0.05)$ and elevated Hif- $1 \alpha$ expression $(P=0.06)$ as independent predictors of OS (Table 2$)$. Median OS for patients with SSTR-2-negative tumours was 5.7 years (95\% CI 3.9-7.5) compared with 10.3 months (95\% CI 0.3-20.4, Log-rank $P=0.03$ ) of the SSTR-2 positive cases (Figure 2B). Patient with Hif- $1 \alpha$ overexpressing tumours had a median OS of 5.8 years $(95 \%$ CI 3.6-7.9) compared with 9.6 median OS (95\% CI 5.2-14.0, Logrank $P=0.03$ ) of samples with low Hif- $1 \alpha$ expression (Figure $2 \mathrm{C}$ ).

On the basis of the results of the multivariate screening of prognostic biomarkers, we combined Hif- $1 \alpha$ overexpression and SSTR-2 expression loss as individual categories with equal weighting to devise a composite prognostic signature. Briefly, patients received a score of 1 for either SSTR-2 loss or Hif- $1 \alpha$ overexpression, resulting in a model with three different the prognostic strata $(0,1$ and 2$)$. Median survival for patients with a score of 0 was not reached over a median follow up time of 8.8 years. Patients with a score of 1 had a median OS of 9.5 years $(95 \%$ CI 5.0-14.0), whereas patient allocated a score of 2 had a median OS of only 4.2 (95\% CI 2.1-6.3, Log-rank $P=0.006$ ) (Figure 3). When tested on an independent multivariate Cox regression model using a backwards elimination approach, the newly devised prognostic signature predicted for patients' OS with a multivariate hazard ratio (HR) of 2.3 (95\% CI 1.0-5.4, $P=0.04$ ), independently from tumour stage (HR 2.3, 95\% CI 1.1-5.4, $P=0.04$ ) and WHO grading $(P=0.23)$.

\section{DISCUSSION}

Prediction of the biological behaviour of NETs is currently based on tumour cell differentiation and Ki-67 as a marker of proliferation (Kloppel, 2011). These are not always reliable markers of prognosis and there is an acute need for better prognostic markers. As endocrine tumours are highly vascular, markers of angiogenesis and its drivers warrant investigation in terms of their prognostic significance. In this paper, we evaluated selected biomarkers of hypoxia and angiogenesis together with SSTR expression in a consecutive, unselected series of GEP-NETs.

We have identified a novel immunohistochemical expression signature emerging from the multivariate screening of prognostic

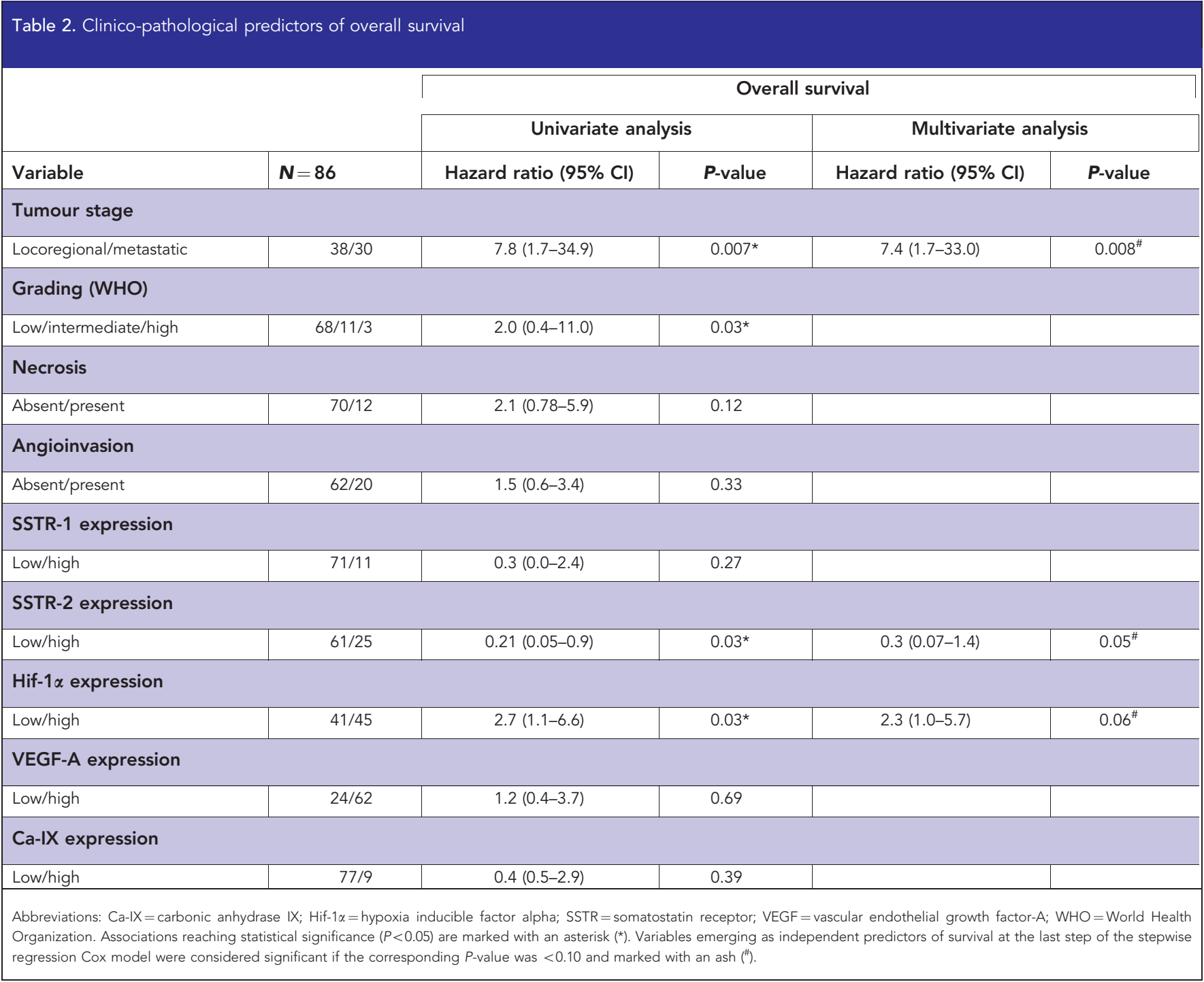




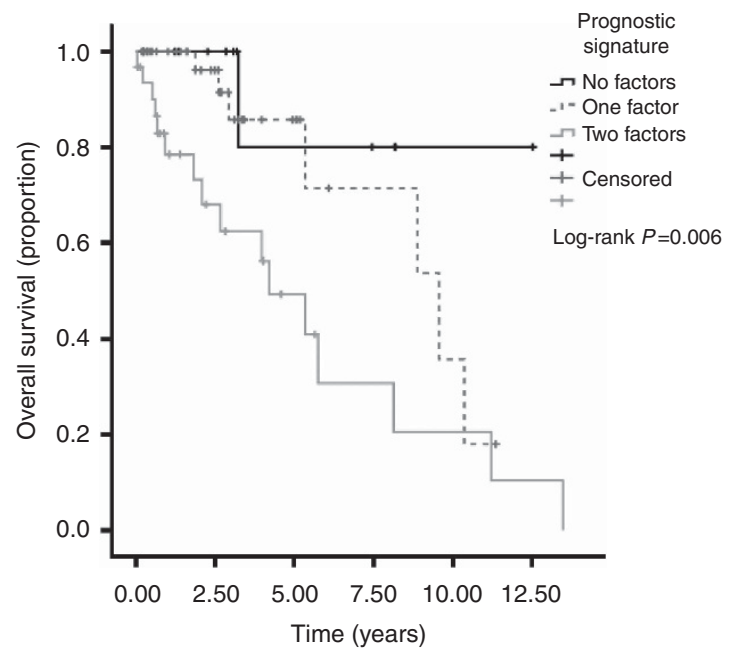

Figure 3. Derivation of an immunohistochemical prognostic signature in GEPNETs based on Hif- $1 \alpha$ and SSTR-2 expression status. KaplanMeier survival curves show that for patients with preserved SSTR-2 and low Hif- $1 \alpha$ (i.e., no adverse prognostic factors, black line) median OS was not reached after a median follow up of 8.8 years. Patients with SSTR-2 loss and high Hif- $1 \alpha$ expression (i.e., two adverse prognostic factors, grey dotted line) had a median survival of only 4.2 years. Patients with only one adverse prognostic factor (either SSTR-2 loss or elevated Hif-1 $\alpha$ expression, grey continuous line) had a median OS of $\sim 10$ years from diagnosis.

tissue biomarkers using a TMA approach. We show that the combined expression status of SSTR- 2 and Hif- $1 \alpha$ can shape a three-tiered prognostic system with a highly significant difference in median survival times for each category of patients. Interestingly, the prognostic power of the expression signature is independent from other commonly used predictors of outcome such as tumour staging or grading.

Somatostatin receptor signalling pathways can promote a wide range of downstream effects in GEP-NETs including inhibition of cell secretion and growth through the modulation of mitogenactivated protein kinase (MAPK) signalling or induction of apoptosis (Patel and Srikant, 1997). Our study reproduces the observation made by Kim et al (2011), where SSTR-2 expression predicted for better survival outcomes in a large series of GEPNETs. This finding may be a reflection of tumour grade, with loss of SSTR-2 expression being a feature of less-differentiated tumours, mirroring the association between the lack of uptake of radiolabelled somatostatin analogues in poorly differentiated tumours (Ezziddin et al, 2006).

The relevance of SSTR-2 expression may, however, extend beyond its prognostic value in GEP-NETs and may potentially influence the use of somatostatin analogue therapy. The somatostatin analogue octreotide binds preferentially to SSTR-2 (Kim et al, 2011). Previous studies have shown that octreotide administration results in tumour response and improved OS in patients with liver metastases from carcinoid tumours (Rinke et al, 2009) and that patients with low-grade tumours and preserved SSTR-2 and 5 expressions have better survival times while on somatostatin analogues (Corleto et al, 2009). In future studies it would be of interest to assess whether SSTR-2 expression does correlate to outcome in patients treated with octreotide.

Recent evidence has shown interplay between SSTR signalling and hypoxia, suggesting that the therapeutic efficacy of octreotide may be at least, in part, explained by the downregulation of Hif- $1 \alpha$ transcriptional activity (Villaume et al, 2010). Hypoxia inducible factor $1 \alpha$, a master regulator of the hypoxic response, is a transcription factor that controls the expression of downstream proteins including, among the others, VEGF-A and Ca-IX, whose role is crucial in securing survival advantage of tumour cells in hypoxic conditions by promoting neovascular supply and reducing intracellular acidosis (Giatromanolaki and Harris, 2001). Although the importance of hypoxia has been studied in a number of tumour types there has been a paucity of studies considering hypoxia in GEP-NETs except in patients with pancreatic NETs arising in a background of VHL syndrome. In this patient group, a strong correlation has been demonstrated between HIF-1 $\alpha$, Ca-IX and VEGF expression (Perigny et al, 2009; O’Toole et al, 2010). Interestingly, although Hif- $1 \alpha$ seems central in the progression of pancreatic NETs, where the activation of the Hif- $1 \alpha / \mathrm{Ca}-\mathrm{IX}$ transcriptional programme associates with poor prognosis, the expression of VEGF negatively correlates with tumour progression (Couvelard et al, 2005). In our study, we identified Hif- $1 \alpha$ but not Ca-IX expression as a predictor of OS. Such difference with the Couvelard study may be explained by the inherent differences in the patient populations considered, including differing sample size, the inclusion of patients with VHL disease as well as the lack of multivariate screening of prognostic variables.

Interestingly, the pathogenic role of hypoxia is confirmed in non-pancreatic GI-NETs, where Hif- $1 \alpha$ is expressed homogenously in both primaries and metastases (Arvidsson et al, 2010). In our study, the majority of tumours displayed evidence of VEGF-A expression, in keeping with the concept that GEP-NETs are highly vascular tumours (Terris et al, 1998; Seo et al, 2000; Zhang et al, 2007; Arvidsson et al, 2010). Despite finding that both VEGF-A and Hif- $1 \alpha$ expressions correlated with the presence of liver metastases, we did not demonstrate any association between VEGF-A and OS. There is a lack of consensus in the literature regarding the prognostic role of VEGF expression in NETs, with some studies suggesting a prognostic role (Zhang et al, 2007), whereas others refuting this (Takahashi et al, 2007; Kuiper et al, 2011). Moreover, in pancreatic NETs, VEGF-A expression and microvascular count seem to paradoxically reduce with progressive tumour de-differentiation (Scoazec, 2012) such that NETs with lower microvessel density have a worse outcome (Marion-Audibert et al, 2003). This may highlight a more complex functional role for the VEGF family, where certain isoforms retain antivascular properties (Albrecht et al, 2010). Another factor that may account for the lack of association between VEGF-A levels and survival in our study may be the relatively good prognosis of patients with GEP-NETs, where 5-year survival figures range between 25 and $100 \%$ (Metz and Jensen, 2008; Modlin et al, 2008) even in the presence of liver metastases. As the median survival in our cohort was 8.8 years, with the majority of tumours being well differentiated and early stage, we hypothesise that the stage and grade distribution of our cohort may not be adequate to detect a prognostic role for VEGF-A, whose expression seems to be strongly stage and grade-dependant.

We confirmed that WHO grading based on Ki-67 proliferation count was associated with a more aggressive disease course and worse OS, confirming the association between tumour proliferation and prognosis (Strosberg et al, 2009; Dimou et al, 2010; Panzuto et al, 2012). This association translates into the clinical recommendation of using Ki-67 expression to guide the administration of systemic chemotherapy, based on the evidence that treatmentinduced benefits are minimal in tumours with Ki-67 index $<1 \%$ (O’Toole et al, 2010).

In conclusion, we have adopted a multivariate screening process of tissue biomarkers to identify novel predictors of outcome relating to hypoxia, angiogenesis and SSTR signalling. We have shown that differential expression of SSTR-2 is predictive of OS in patients with NETs, and that the presence of hypoxia as measured by Hif- $1 \alpha$ overexpression are potential predictors of clinical outcome in patients with GEP-NETs. 
We used this information to generate a coherent immunohistochemical expression signature that combines the independent prognostic information obtained from SSTR-2 status and Hif- $1 \alpha$ overexpression, and confirmed that this signature accurately predicts prognosis independent of tumour stage and proliferation status, allowing us to further refine survival prediction in a patient population that is generally regarded as carrying a good prognosis. We are limited by the need for a prolonged time span used for patient accrual, during which radical changes in diagnosis and management may have influenced survival outcomes. This was dictated by the relative low incidence rates of GEP-NETs and the long survival times that characterise the natural history of these tumours and is a common limitation applying to most studies investigating prognostic factors in this tumour type. In addition, it would be important to ascertain whether our newly identified prognostic signature can predict progression-free survival, a more easily quantifiable survival endpoint in cancers with a relatively indolent biological behaviour.

In future studies, it would be important to validate our findings in an independent, larger patient cohort, considering treatment regimens administered, which was incomplete in our cohort.

\section{ACKNOWLEDGEMENTS}

We acknowledge the contribution of Lynn Maslen, who accurately reviewed the manuscript before submission. We are indebted to Marco Vergine for his histopathological expertise. We also acknowledge Tyler Lloyd's contribution for having helped to optimise the antibodies used for this project.

\section{CONFLICT OF INTEREST}

The authors declare no conflict of interest.

\section{REFERENCES}

Albrecht I, Kopfstein L, Strittmatter K, Schomber T, Falkevall A, CE Hagberg, Lorentz P, Jeltsch M, Alitalo K, Eriksson U, Christofori G, Pietras K (2010) Suppressive effects of vascular endothelial growth factor-B on tumor growth in a mouse model of pancreatic neuroendocrine tumorigenesis. PLoS One 5(11): e14109.

Arvidsson Y, Bergstrom A, Arvidsson L, Kristiansson E, Ahlman H, Nilsson O (2010) Hypoxia stimulates CXCR4 signalling in ileal carcinoids. Endocr Relat Cancer 17(2): 303-316.

Chi JT, Wang Z, Nuyten DS, Rodriguez EH, Schaner ME, Salim A, Wang Y, Kristensen GB, Helland A, Borresen-Dale AL, Giaccia A, Longaker MT, Hastie T, Yang GP, van de Vijver MJ, Brown PO (2006) Gene expression programs in response to hypoxia: cell type specificity and prognostic significance in human cancers. PLoS Med 3(3): e47.

Corleto VD, Falconi M, Panzuto F, Milione M, De Luca O, Perri P, Cannizzaro R, Bordi C, Pederzoli P, Scarpa A, Delle Fave G (2009) Somatostatin receptor subtypes 2 and 5 are associated with better survival in well-differentiated endocrine carcinomas. Neuroendocrinology 89(2): 223-230.

Couvelard A, O’Toole D, Turley H, Leek R, Sauvanet A, Degott C, Ruszniewski P, Belghiti J, AL Harris, Gatter K, Pezzella F (2005) Microvascular density and hypoxia-inducible factor pathway in pancreatic endocrine tumours: negative correlation of microvascular density and VEGF expression with tumour progression. Br J Cancer 92(1): 94-101.

Dal Monte M, Cammalleri M, Martini D, Casini G, Bagnoli P (2007) Antiangiogenic role of somatostatin receptor 2 in a model of hypoxiainduced neovascularization in the retina: results from transgenic mice. Invest Ophthalmol Vis Sci 48(8): 3480-3489.

Dimou AT, Syrigos KN, Saif MW (2010) Neuroendocrine tumors of the pancreas: what's new. Highlights from the ' 2010 ASCO Gastrointestinal Cancers Symposium'. Orlando, FL, USA. January 22-24, 2010. JOP 11(2): 135-138.
Ezziddin S, Logvinski T, Yong-Hing C, Ahmadzadehfar H, HP Fischer, Palmedo H, Bucerius J, MJ Reinhardt, HJ Biersack (2006) Factors predicting tracer uptake in somatostatin receptor and MIBG scintigraphy of metastatic gastroenteropancreatic neuroendocrine tumors. J Nucl Med 47(2): 223-233.

Giatromanolaki A, Harris AL (2001) Tumour hypoxia, hypoxia signaling pathways and hypoxia inducible factor expression in human cancer. Anticancer Res 21(6B): 4317-4324.

Hanahan D, Weinberg RA (2000) The hallmarks of cancer. Cell 100(1): 57-70.

Hirabayashi K, Zamboni G, Nishi T, Tanaka A, Kajiwara H, Nakamura N (2013) Histopathology of gastrointestinal neuroendocrine neoplasms. Front Oncol 3: 2.

Hui EP, Chan AT, Pezzella F, Turley H, To KF, Poon TC, Zee B, Mo F, Teo PM, Huang DP, Gatter KC, Johnson PJ, Harris AL (2002) Coexpression of hypoxia-inducible factors 1alpha and 2alpha, carbonic anhydrase IX, and vascular endothelial growth factor in nasopharyngeal carcinoma and relationship to survival. Clin Cancer Res 8(8): 2595-2604.

Katoh R (2003) Angiogenesis in endocrine glands: special reference to the expression of vascular endothelial growth factor. Microsc Res Tech 60(2): 181-185.

Kim HS, Lee HS, Kim WH (2011) Clinical significance of protein expression of cyclooxygenase- 2 and somatostatin receptors in gastroenteropancreatic neuroendocrine tumors. Cancer Res Treat 43(3): 181-188.

Kloppel G (2011) Classification and pathology of gastroenteropancreatic neuroendocrine neoplasms. Endocr Relat Cancer 18(Suppl 1): S1-16.

Kuiper P, Hawinkels LJ, De Jonge-Muller ES, Biemond I, Lamers CB, Verspaget HW (2011) Angiogenic markers endoglin and vascular endothelial growth factor in gastroenteropancreatic neuroendocrine tumors. World J Gastroenterol 17(2): 219-225.

LeCouter J, Kowalski J, Foster J, Hass P, Zhang Z, Dillard-Telm L, Frantz G, Rangell L, DeGuzman L, GA Keller, Peale F, Gurney A, KJ Hillan, Ferrara N (2001) Identification of an angiogenic mitogen selective for endocrine gland endothelium. Nature 412(6850): 877-884.

Marion-Audibert AM, Barel C, Gouysse G, Dumortier J, Pilleul F, Pourreyron C, Hervieu V, Poncet G, Lombard-Bohas C, Chayvialle JA, Partensky C, Scoazec JY (2003) Low microvessel density is an unfavorable histoprognostic factor in pancreatic endocrine tumors. Gastroenterology 125(4): 1094-1104.

Maynard MA, Ohh M (2005) Molecular targets from VHL studies into the oxygen-sensing pathway. Curr Cancer Drug Targets 5(5): 345-356.

Mentlein R, Eichler O, Forstreuter F, Held-Feindt J (2001) Somatostatin inhibits the production of vascular endothelial growth factor in human glioma cells. Int J Cancer 92(4): 545-550.

Metz DC, Jensen RT (2008) Gastrointestinal neuroendocrine tumors: pancreatic endocrine tumors. Gastroenterology 135(5): 1469-1492.

Modlin IM, Oberg K, Chung DC, Jensen RT, De Herder WW, Thakker RV, Caplin M, Delle Fave G, Kaltsas GA, Krenning EP, Moss SF, Nilsson O, Rindi G, Salazar R, Ruszniewski P, Sundin A (2008) Gastroenteropancreatic neuroendocrine tumours. Lancet Oncol 9(1): 61-72.

O’Toole D, Couvelard A, Rebours V, Zappa M, Hentic O, Hammel P, Levy P, Bedossa P, Raymond E, Ruszniewski P (2010) Molecular markers associated with response to chemotherapy in gastro-entero-pancreatic neuroendocrine tumors. Endocrine-Related Cancer 17(4): 847-856.

Panzuto F, Campana D, Fazio N, MP Brizzi, Boninsegna L, Nori F, Di Meglio G, Capurso G, Scarpa A, Dogliotti L, De Braud F, Tomassetti P, Delle Fave G, Falconi M (2012) Risk factors for disease progression in advanced jejunoileal neuroendocrine tumors. Neuroendocrinology 96(1): $32-40$.

Patel YC, Srikant CB (1997) Somatostatin receptors. Trends Endocrinol Metab 8(10): 398-405.

Perigny M, Hammel P, Corcos O, Larochelle O, Giraud S, Richard S, Sauvanet A, Belghiti J, Ruszniewski P, Bedossa P, Couvelard A (2009) Pancreatic endocrine microadenomatosis in patients with von HippelLindau disease: characterization by VHL/HIF pathway proteins expression. Am J Surg Pathol 33(5): 739-748.

Pinato DJ, Mauri FA, Ramakrishnan R, Wahab L, Lloyd T, Sharma R (2012a) Inflammation-based prognostic indices in malignant pleural mesothelioma. J Thorac Oncol 7(3): 587-594.

Pinato DJ, Ramachandran R, Toussi ST, Vergine M, Ngo N, Sharma R, Lloyd T, Meeran K, Palazzo F, Martin N, Khoo B, Dina R, Tan TM (2012b) Immunohistochemical markers of the hypoxic response can identify malignancy in phaeochromocytomas and paragangliomas and 
optimize the detection of tumours with VHL germline mutations. Br J Cancer 108: 429-437.

Raymond E, Dahan L, JL Raoul, YJ Bang, Borbath I, Lombard-Bohas C, Valle J, Metrakos P, Smith D, Vinik A, JS Chen, Horsch D, Hammel P, Wiedenmann B, Van Cutsem E, Patyna S, DR Lu, Blanckmeister C, Chao R, Ruszniewski P (2011) Sunitinib malate for the treatment of pancreatic neuroendocrine tumors. N Engl J Med 364(6): 501-513.

Rinke A, HH Muller, Schade-Brittinger C, KJ Klose, Barth P, Wied M, Mayer C, Aminossadati B, UF Pape, Blaker M, Harder J, Arnold C, Gress T, Arnold R (2009) Placebo-controlled, double-blind, prospective, randomized study on the effect of octreotide LAR in the control of tumor growth in patients with metastatic neuroendocrine midgut tumors: a report from the PROMID Study Group. J Clin Oncol 27(28): 4656-4663.

Ruscica M, Arvigo M, Steffani L, Ferone D, Magni P (2012) Somatostatin, somatostatin Analogs and somatostatin receptor dynamics in the biology of cancer progression. Curr Mol Med 13: 555-571.

Scoazec JY (2012) Angiogenesis in neuroendocrine tumors: therapeutic applications. Neuroendocrinology 97: 45-56.

Seo Y, Baba H, Fukuda T, Takashima M, Sugimachi K (2000) High expression of vascular endothelial growth factor is associated with liver metastasis and a poor prognosis for patients with ductal pancreatic adenocarcinoma. Cancer 88(10): 2239-2245.

Strosberg J, Gardner N, Kvols L (2009) Survival and prognostic factor analysis of 146 metastatic neuroendocrine tumors of the mid-gut. Neuroendocrinology 89(4): 471-476.

Takahashi Y, Akishima-Fukasawa Y, Kobayashi N, Sano T, Kosuge T, Nimura Y, Kanai Y, Hiraoka N (2007) Prognostic value of tumor architecture, tumor-associated vascular characteristics, and expression of angiogenic molecules in pancreatic endocrine tumors. Clin Cancer Res 13(1): 187-196.

Terris B, JY Scoazec, Rubbia L, Bregeaud L, MS Pepper, Ruszniewski P, Belghiti J, Flejou J, Degott C (1998) Expression of vascular endothelial growth factor in digestive neuroendocrine tumours. Histopathology 32(2): 133-138.

Villaume K, Blanc M, Gouysse G, Walter T, Couderc C, Nejjari M, Vercherat C, Cordier-Bussat M, Roche C, JY Scoazec (2010) VEGF secretion by neuroendocrine tumor cells is inhibited by octreotide and by inhibitors of the PI3K/AKT/mTOR pathway. Neuroendocrinology 91(3): 268-278.

Yao JC, Hassan M, Phan A, Dagohoy C, Leary C, Mares JE, Abdalla EK, Fleming JB, Vauthey JN, Rashid A, Evans DB (2008) One hundred years after 'carcinoid': epidemiology of and prognostic factors for neuroendocrine tumors in 35825 cases in the United States. J Clin Oncol 26(18): 3063-3072.

Zhang J, Jia Z, Li Q, Wang L, Rashid A, Zhu Z, DB Evans, JN Vauthey, Xie K, JC Yao (2007) Elevated expression of vascular endothelial growth factor correlates with increased angiogenesis and decreased progression-free survival among patients with low-grade neuroendocrine tumors.

Cancer 109(8): 1478-1486.

This work is published under the standard license to publish agreement. After 12 months the work will become freely available and the license terms will switch to a Creative Commons AttributionNonCommercial-Share Alike 3.0 Unported License. 